\title{
Virally mediated MafB transduction induces the monocyte commitment of human CD34 + hematopoietic stem/progenitor cells
}

\author{
C Gemelli ${ }^{1}$, M Montanari ${ }^{1}$, E Tenedini ${ }^{1}$, T Zanocco Marani ${ }^{1}$, \\ T Vignudelli ${ }^{1}$, M Siena ${ }^{1}$, R Zini $^{1}$, S Salati ${ }^{1}$, E Tagliafico ${ }^{1}$, \\ R Manfredini ${ }^{1}$, A Grande ${ }^{1,2}$ and S Ferrari, ${ }^{*, 1,2}$ \\ ${ }^{1}$ Department of Biomedical Sciences, University of Modena and Reggio Emilia, \\ Modena, Italy \\ ${ }^{2}$ These authors share the senior authorship \\ * Corresponding author: S Ferrari, Dipartimento di Scienze Biomediche, \\ Sezione di Chimica Biologica, Università di Modena e Reggio Emilia, Via \\ Campi 287, 41100 Modena, Italy. Tel: + 39-059-2055400; \\ Fax + 39-059-2055410; E-mail: sergio@unimo.it
}

Received 01.8.05; revised 07.12.05; accepted 07.12.05; published online 03.2.06 Edited by $\mathrm{G}$ Cossu

\begin{abstract}
Upregulation of specific transcription factors is a generally accepted mechanism to explain the commitment of hematopoietic stem cells along precise maturation lineages. Based on this premise, transduction of primary hematopoietic stem/ progenitor cells with viral vectors containing the investigated transcription factors appears as a suitable experimental model to identify such regulators. Although MafB transcription factor is believed to play a role in the regulation of monocytic commitment, no demonstration is, to date, available supporting this function in normal human hematopoiesis. To address this issue, we retrovirally transduced cord blood CD34 + hematopoietic progenitors with a MafB cDNA. Immunophenotypic and morphological analysis of transduced cells demonstrated the induction of a remarkable monomacrophage differentiation. Microarray analysis confirmed these findings and disclosed the upregulation of macrophage-related transcription factors belonging to the AP-1, MAF, PPAR and MiT families. Altogether our data allow to conclude that MafB is a key regulator of human monocytopoiesis.

Cell Death and Differentiation (2006) 13, 1686-1696.

doi:10.1038/sj.cdd.4401860; published online 3 February 2006
\end{abstract}

Keywords: MafB; monocyte commitment; CD34 + cells

Abbreviations: HSC, hematopoietic stem cell; $\mathrm{CB}$, cord blood; MARE, Maf responsive element; IMDM, Iscove's modified Dulbecco's medium; SCF, stem cell factor; Flt3-I, Flt3-ligand; TPO, thrombopoietin; IL, interleukin; PB, peripheral blood; Mo, monoclonal; $\mathrm{Ab}$, antibody; VD, $1 \alpha, 25$ di-hydroxy-vitamin $\mathrm{D}_{3}$; FACS, fluorescence activated cell sorter; RPE-Cy5, R-Phycoerythrin-Cy5; FITC, fluorescein isothiocyanate; PE, phycoerythrin; $\mathrm{BFU}-\mathrm{E}$, burst forming unit-erythroid; CFU-E, colony forming uniterythroid; CFU-GM, colony forming unit-granulocyte/monocyte; CFU-G, colony forming unit-granulocyte; CFU-M, colony forming unit-monocyte; GAPDH, glyceraldehydes 3-phospate dehydrogenase; SDS, sodium dodecyl sulphate; MAS 5.0, microarray analysis suite 5.0; P, present; M, marginal; A, absent; I, increased; D, decreased; NC, not changed; SLR, signal log ratio; LTR, long terminal repeats; $\triangle \mathrm{INGFR}$, truncated low-affinity nerve growth factor receptor; BrdU, bromodeoxyuridine. All genes cited in the microarray data section were named by gene symbols: see the OMIM website ( www.ncbi.nlm.nih.gov) for extended denomination and/or further explanation

\section{Introduction}

Although hematopoiesis is a complex process, it can be schematically divided into two sequential steps. In the initial 'commitment' (or 'lineage decision') phase, pluripotent hematopoietic stem cells (HSCs) undergo a gradual restriction of their differentiation potential, eventually resulting in the production of unipotent hematopoietic progenitors/precursors. ${ }^{1,2}$ During the subsequent 'terminal differentiation' phase, these last cells give rise to all the mature blood cells, characterized by different phenotypes and specialized functions. ${ }^{1,2}$ A large number of reports indicate that commitment of HSCs along precise differentiation lineages is the consequence of spontaneous or exogenously induced upregulation of specific transcription factors. ${ }^{3,4}$ In this regard, a clear distinction should be established between transcription factors that are generically involved in the molecular control of hematopoietic cell differentiation and the so-called 'master' regulator transcription factors, which are by themselves able to promote the lineage commitment of pluripotent HSCs or the lineage 'switching' of committed progenitors when introduced into a variety of primary cells (embrionic or HSCs, transformed multipotent hematopoietic progenitors). ${ }^{3-5}$ Based on this approach, several transcription factors have been demonstrated to behave as 'master' (or 'key') regulators in the complex process of lineage commitment decision, the most important being PAX5 for B lymphopoiesis, GATA-1 for erythroid differentiation, $\mathrm{PU}-1$ for myeloid commitment and $\mathrm{C}-\mathrm{EBP} \alpha$ (and other C-EBP family members) for granulocytic differentiation. ${ }^{4}$ Similarly, the HOX-A10 gene was shown to trigger the mono-macrophage commitment of cord blood (CB) $\mathrm{CD} 34+$ hematopoietic progenitors ${ }^{6}$ and the MafB transcription factor was demonstrated to promote the monocytic differentiation of Myb-Ets transformed avian hematopoietic progenitors. ${ }^{7}$ MafB is a member of the Maf basic region/ leucine zipper transcription factor family. ${ }^{8}$ This family is divided into two subgroups: the large and the small Maf proteins. $^{8}$ All these proteins share a common carboxyterminal portion bearing a basic region/leucine zipper domain mediating DNA binding and homo- and hetero-dimer assembly. ${ }^{8}$ The large Maf proteins (c-Maf, MafB, NRL, L-Maf, MafA) contain an acidic amino-terminal transactivation domain that 
is lacking in the small proteins (MafK, MafF, MafG). ${ }^{8}$ Regardless of the presence of a transactivation domain, all Maf family members display binding activity to a common DNA sequence (MARE), and can either activate or repress transcription depending on the interacting dimerization partner. ${ }^{8}$ Both homo- and hetero-dimer formation have been described although these last complexes can only be established among members of the same sub-family (large or small) or with non-Maf transcription factors. ${ }^{8}$ In general, Maf proteins are ubiquitously expressed and involved in the regulation of a number of biological processes like tumorigenesis, development and differentiation. ${ }^{8}$ Several members of the Maf family have been demonstrated to play a role in hematopoiesis and, besides the above mentioned MafB protein, at least another member, namely c-Maf, was shown to induce the mono-macrophagic differentiation of myeloid precursors although this observation was exclusively obtained in cell line models. ${ }^{9}$ Additionally, experiments of gene expression profiling performed in our laboratory showed that MafB is highly and specifically expressed in CD34 + derived monocyte precursors as compared to granulocyte precursors. ${ }^{10}$ In spite of the evidence that MafB behaves as a determinant of monocytic commitment in transformed avian hematopoietic progenitors, ${ }^{7}$ a clear demonstration of the possible role played by this transcription factor in human normal monocytopoiesis is, to date, still lacking. To address this issue, we retro-virally transduced U937 and THP1 leukemic monoblasts and CB CD34 + hematopoietic stem/ progenitor cells with a 'full-length' MafB cDNA. Morphological, immunopenotypic and microarray analysis of transduced cells indicated that MafB determines a massive induction of monomacrophage differentiation accompanied by a dramatic inhibition of erythroid commitment, indicating that this transcription factor can be considered a master regulator of monocytic commitment in human hematopoiesis.

\section{Results}

\section{Endogenous MafB expression in hematopoietic cells}

To identify genes associated with monocyte differentiation, we performed a clustering analysis on microarray data obtained from seven normal hematopoietic cell populations of human origin and more precisely: CD34 + progenitors, monoblasts, monocytes, myeloblasts, neutrophils, erythroblasts and megakaryoblasts. For this purpose, we used the GeneSpring software to sort, from the different analyzed expression profiles, genes that were more than three-fold induced in monoblasts/monocytes and less than three-fold induced in the other cells types as compared to CD34 + cells. By using this procedure we could identify 86 genes exhibiting a monocyte related expression pattern. Results of this analysis, reported in Figure 1 as Eisen map, evidenced that MafB was over 15-fold more expressed in monoblasts/monocytes in comparison with CD34+ cells and, with the only exception of EGR2, no other transcription factors were detectable among these monocyte related genes. In other terms, clustering analysis allowed to conclude that MafB mRNA is highly and specifically expressed in

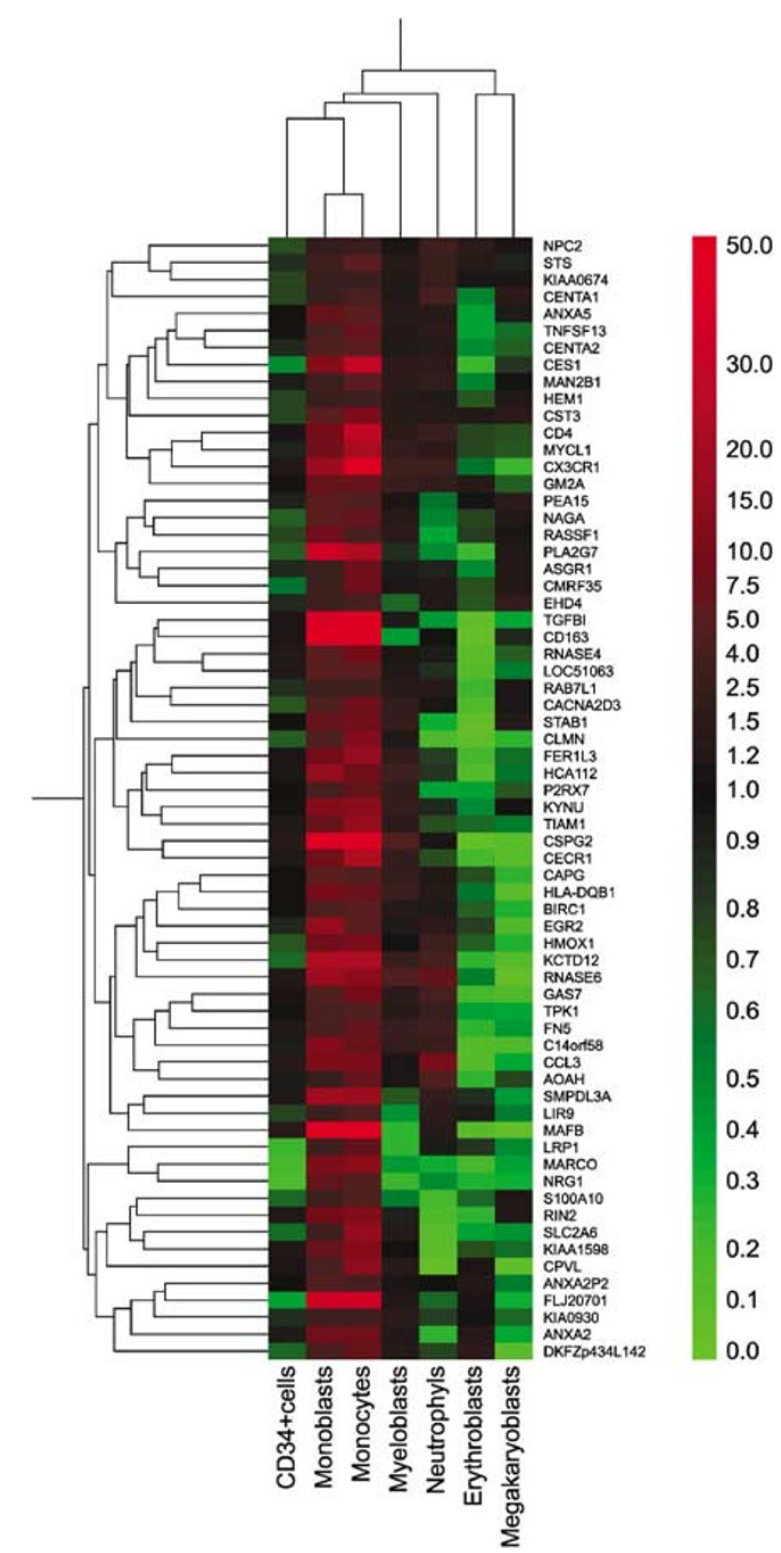

Figure 1 Clustering analysis performed on expression profiles of seven normal hematopoietic cell populations to identify genes related to the monocytic context. GeneSpring software was used to identify genes that were more than three-fold induced in monoblasts/monocytes and less than three-fold induced in the other cells types as compared to CD34 + cells. Sorted genes are presented as Eisen map obtained by using the 'gene tree' and 'condition tree' clustering algorythms. Analyzed cell populations are reported in the bottom. Genes are indicated by Gene Symbols and reported on the right of the Eisen map (extended gene designation and description are available in the OMIM website). The signal based coloring scale is also shown on the right hand side of the figure

monoblasts and monocytes as compared to nonmonocytic cell populations.

Semiquantitative RT-PCR analysis substantially confirmed these data, revealing that MafB expression is barely detectable in CD34+ stem/progenitor cells, granulocytes and lymphocytes, whereas it is strongly induced in mature circulating monocytes (Figure 2, left panel). Consistently with these findings, MafB expression was virtually restricted to hematopoietic cell lines having a monocyte precursor 


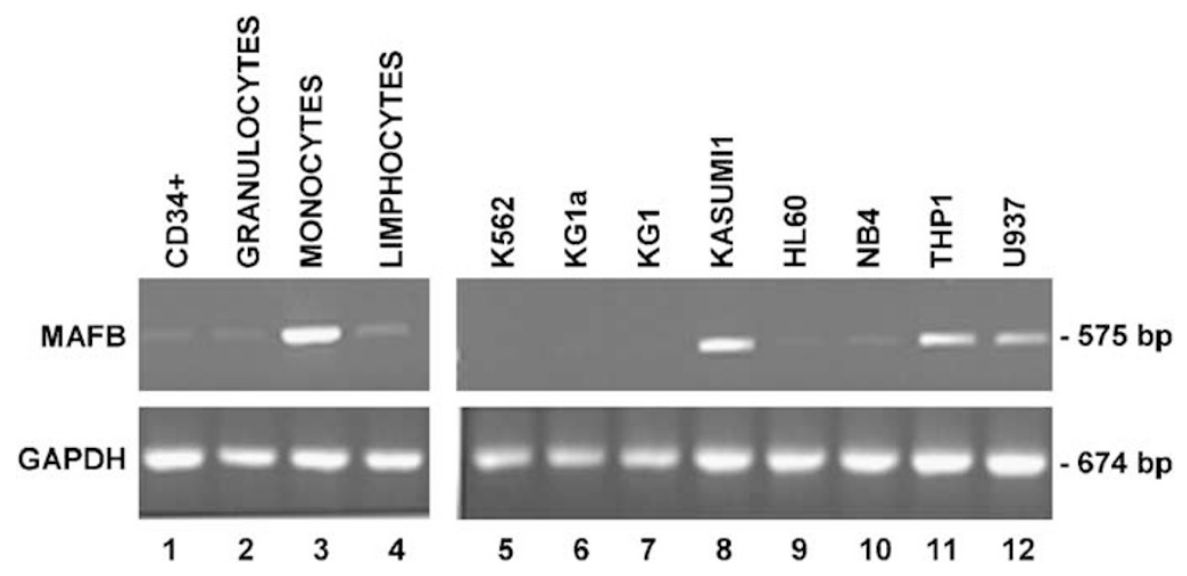

Figure 2 MafB mRNA expression in normal and leukemic hematopoietic cell populations. Expression levels of MafB mRNA were assessed by semiquantitative RTPCR analysis performed on: normal cord blood CD34 + stem/progenitor cells, adult peripheral blood granulocytes, monocytes and lymphocytes (left panel) and hematopoietic cell lines having different phenotype (right panel). GAPDH mRNA was also amplified to normalize RNA amounts of the different analyzed samples. Agarose gels stained with ethidium bromide are shown. The size of amplified gene fragments is reported on the right hand side of each panel

phenotype (Kasumi1, THP1, U937) (Figure 2, right panel). Taken together these results demonstrate that, at least in hematopoietic cells, MafB expression is monocyte-specific and maturation stage-related suggesting a probable involvement in the transcriptional regulation of monocytic commitment.

\section{Retroviral vector mediated expression of a MafB full-length cDNA in monoblastic cell lines}

To address this issue, we constructed the $L M a f B I \Delta N$ retroviral vector expressing a MafB full-length $\mathrm{CDNA}$ and a truncated version of low-affinity Nerve Growth Factor Receptor ( $\triangle$ LNGFR), used as 'reporter' gene, in the context of a bicistronic transcript driven by the viral LTR (Supplementary Figure 1a). This vector was initially used to transduce the U937 and THP1 hematopoietic cell lines, due to their monoblastic phenotype and responsivity to compounds inducing mono-macrophage differentiation. Biological effects promoted by the MafB containing vector were assessed by comparison with untransduced cells cultured in the same well or flask, therefore maintained under the same experimental conditions, and subsequently analyzed upon immuno-magnetic separation or flow cytometry gating based on NGFR positivity. To exclude any interference of the $\triangle \mathrm{LNGFR}$ 'reporter' gene with the analyzed processes, cells transduced with the LXI $\Delta N$ 'empty' vector were also used as control.

Infection of U937 cells with the LMafBI $\Delta \mathrm{N}$ retroviral vector resulted in a $35 \pm 5 \%$ gene transfer efficiency as monitored by flow cytometry. Northern and Western blot analysis, performed on transduced/NGFR purified cells, respectively evidenced that the retroviral transcript was regularly produced (Supplementary Figure 1b) and the transgene was highly expressed at the protein level (Supplementary Figure 1c), thus mimicking the expression pattern observed in hematopoietic cells (see Figures 1 and 2). Flow cytometry examination of monocyte differentiation markers, in MafB transduced cells, revealed a gradual increase of CD11b positivity up to $25 \%$ reached at day 14 after infection (Figure $3 a$ ) and, even if

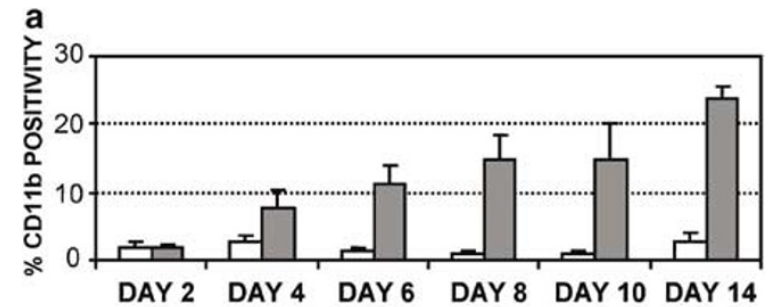

b
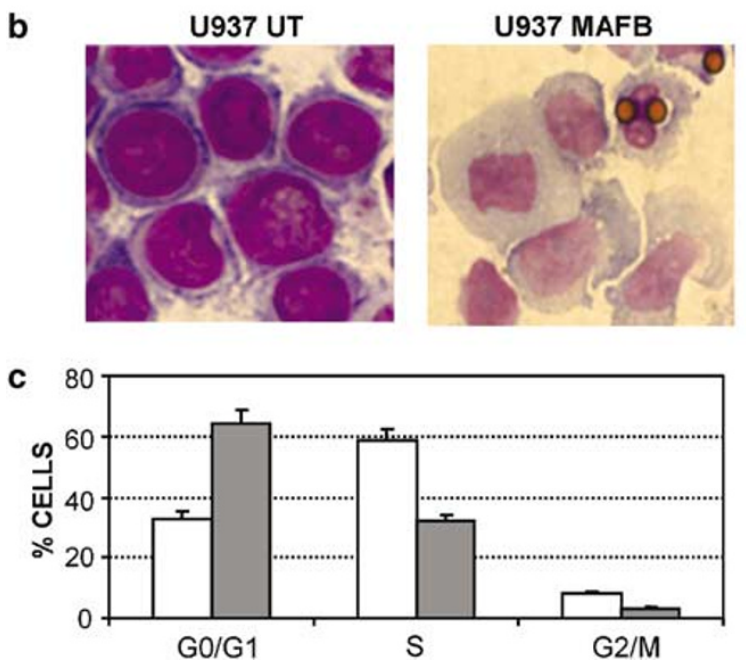

Figure 3 Effect of MafB transduction in U937 cells. (a) Histogram reporting the results of flow cytometry analysis of the myeloid related CD11b antigen, measured as positivity percentage, in MafB transduced (gray bars) and untransduced (white bars) U937 cells. Days post-transduction are indicated on $x$ axis. Values are reported as mean \pm S.E.M. (b) Morphological analysis performed 14 days postinfection on untransduced (U937 UT) and MafB transduced/NGFR purified (U937 MAFB) U937 cells. (c) Histogram summarizing cell cycle distribution of MafB transduced (gray bars) as compared to untransduced (white bars) U937 cells. Analysis was performed 10 days after retroviral transduction upon propidium iodide/BrdU staining followed by flow cytometry examination. Values are reported as mean \pm S.E.M.

not detected by immunophenotype assessment, the CD14 antigen underwent a clear induction when analyzed by quantitative real time PCR (not shown). Morphological 


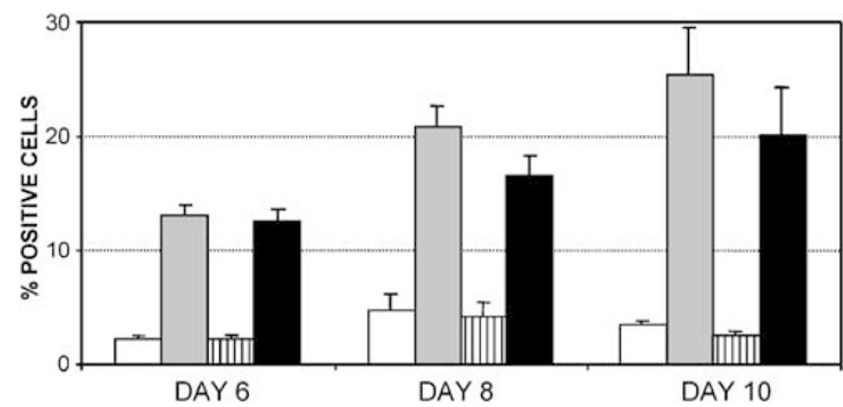

Figure 4 Effect of MafB transduction in THP1 cells. The histogram shows flow cytometry analysis results of the CD14 and CD11b differentiation markers, reported as positivity percentage, in MafB transduced (black and gray bars, respectively) and untransduced (dashed and white bars, respectively) U937 cells. Days posttransduction are indicated on $x$-axis. Data obtained by three independent experiments are reported as average values \pm S.E.M.

analysis demonstrated that a subset of transduced/purified U937 cells $(\sim 45 \%)$ assumed a promonocyte phenotype (Figure $3 \mathrm{~b}$ ) and flow cytometry evaluation of cell cycle distribution, performed upon propidium iodide/BrdU staining, evidenced a remarkable decrease of S-G2/M phase coupled with a consistent increase in the extent of G0/G1 phase (Figure 3c).

The capacity of MafB transcription factor to promote the monocyte differentiation of U937 cells was also confirmed using a distinct monoblastic cell line. In fact, as reported in Figure 4, THP1 leukemic monoblasts transduced with the $\mathrm{LMafBI} \Delta \mathrm{N}$ retroviral vector underwent a comparable differentiation effect characterized by a progressive induction of both CD14 and CD11b surface antigens (25 and 20\%, respectively, at day 10 post-transduction).

Overall, these data indicate that retroviral transduction of MafB induces the monocyte differentiation of U937 and THP1 cells leading, at the same time, to a massive inhibition of cell growth. These biological effects were specifically induced by the $\mathrm{LMafBI} \Delta \mathrm{N}$ retroviral vector and were not observed in untransduced (Figures 3 and 4 ) and $L X I \Delta N$ transduced cells (not shown).

\section{Retroviral expression of MafB cDNA in human CD34 + hematopoietic progenitors}

To verify these data in a primary cell model, we purified $C B$ CD34 + hematopoietic stem/progenitor cells, transduced them with the MafB containing retroviral vector after a 2 days preactivation in liquid culture, and estimated the extent of mono-macrophage differentiation by immune phenotype and morphological analysis. In a set of five independent experiments, gene transfer efficiency, assessed by flow cytometry analysis of $\Delta$ LNGFR positivity, ranged $15-30 \%$. At day 7 of liquid culture, that is to say only 4 days after infection, MafB transduced NGFR + cells exhibited a remarkable induction of the monocyte specific CD14 antigen ${ }^{10}$ as compared to untransduced NGFR - cells ( $53 \pm 4$ versus $12 \pm 2 \%$, respectively) (Figure $5 \mathrm{a}$ and b). CD14 expression remained subsequently stable in transduced cells $(56 \pm 8 \%$ at day 10 and $56 \pm 9 \%$ at day 14 ) showing, on the contrary, a gradual upregulation in untransduced control cells $(25 \pm 2$ and $40 \pm 8 \%$, respectively) (Figure $5 \mathrm{~b}$ ). This result was not surprising and, based on previous observations, it can be clearly ascribed to the capacity of hematopoietic cytokines to induce the spontaneous differentiation of CD34 + cells in liquid culture. ${ }^{11,12}$ Expression of the CD11b myeloid differentiation marker ${ }^{10}$ in MafB transduced cells exhibited a delayed upregulation as compared to CD14 antigen, culminating at day 10 of culture where it raised up to $52 \pm 6$ versus $29 \pm 6 \%$ of control untransduced cells (Figure $5 \mathrm{~b}$ ). Morphological analysis performed at the end of experiment (day 14) evidenced that monocytes-macrophages represented over $90 \%$ of purified NGFR + transduced cells and only $25 \%$ of NGFR - untransduced cells (Figure 5a, right panel). The discrepancy observed between these last data and immunophenotypic analysis performed at day 14 of culture, evidencing a less pronounced differentiation effect in terms of CD14 antigen expression $(56 \pm 9 \%$ of MafB transduced versus $40 \pm 8 \%$ of untransduced cells), is not surprising and reflects the fact that CD14 is downregulated in cultured macrophages $^{10,13}$ and, on the other hand, is transiently expressed in a subset of granulocyte precursors, ${ }^{10}$ that is, cells that are highly represented in control cells under the used experimental conditions (Figure 5a, right panel).

Cells transduced with the $\mathrm{LXI} \Delta \mathrm{N}$ control vector did not differ significantly from untransduced cells in these liquid culture experiments (not shown).

To better characterize the role played by MafB in the commitment phase of hematopoiesis, transduced and NGFR purified CD34 + cells were analyzed by clonogenic assay in methylcellulose performed at day 5 of liquid culture ( 2 days after transduction). The results obtained, reported in Figure 6 , clearly indicated that this transcription factor induces a remarkable increase of monocyte progenitors (CFU-M), from $8.7 \pm 0.8 \%$ of untransduced cells to $39 \pm 2 \%$ of MafB transduced cells, and a dramatic decrease of erythroid progenitors (BFU-E, CFU-E), from $64.8 \pm 1.8$ to $19 \pm 2.4 \%$, respectively. Granulo-monocytic (CFU-GM) and granulocytic (CFU-G) progenitors were not significantly affected by MafB transduction, and, again, cells transduced with the $\mathrm{LXI} \Delta \mathrm{N}$ 'empty' vector exhibited levels of clonogenic progenitors that were comparable to those of the untransduced control. These data indicate that MafB transduction leads to a massive commitment of CD34 + hematopoietic progenitors towards the monocyte differentiation lineage, which is achieved, at least partly, at the expense of other maturation lineages as the erythroid one.

\section{siRNA mediated inactivation of MafB gene in CD34 + progenitors}

To assess the biological effects of MafB gene inactivation in CB CD34 + hematopoietic progenitors, we transfected these cells with an anti-MafB oligonucleotide siRNA by using a standard nucleofection procedure. Differentiation of treated cells was subsequently induced by liquid culture and clonogenic assay performed with the same timings and modalities that have been already described for retrovirally mediated MafB transduction. Despite endogenous MafB 
a
DAY 7

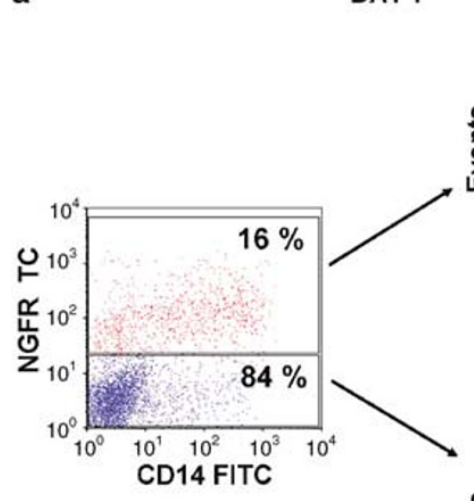

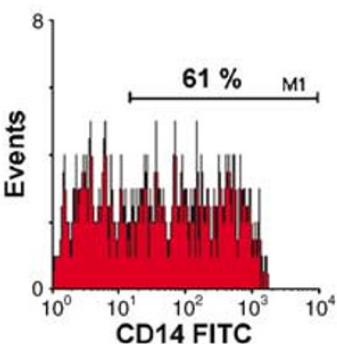

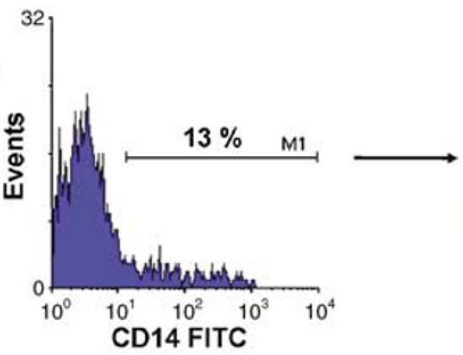

DAY14

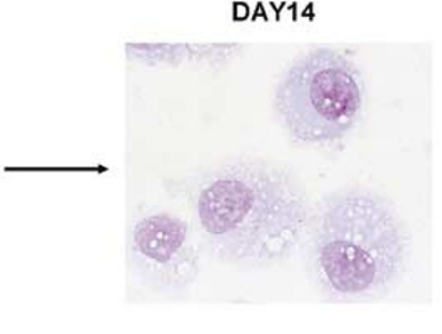

b

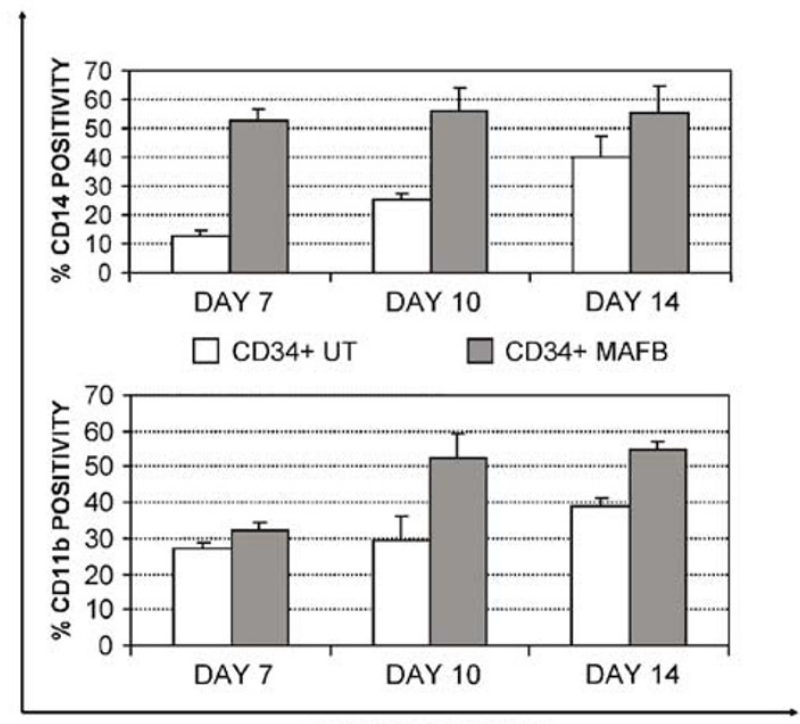

DAYS OF CULTURE

Figure 5 MafB elicited mono-macrophage differentiation of human cord blood CD34 + hematopoietic stem/progenitor cells. CD34 + hematopoietic progenitors were transduced with the $\mathrm{LMafBI} \triangle \mathrm{N}$ retroviral vector and the entity of monocyte differentiation was monitored by flow cytometric and morphological analysis. CD14 and CD11b positivity were estimated on transduced and untransduced control cells 'gated' on the base of their different NGFR expression pattern. Morphological examination was performed on purified and cytocentrifuged NGFR positive and negative cell fractions. (a) CD14 antigen expression (left) and morphological changes (right) observed in a representative experiment at day 7 and 14 of liquid culture, respectively (infection performed at day 3). Histograms reported in (b) summarize flow cytometry analysis results of CD14 and CD11b positivity in MafB transduced (gray bars) and untransduced cells (white bars). Days of culture are indicated on $x$-axis of each histogram. Values are reported as mean \pm S.E.M.

mRNA levels, estimated by quantitative real-time RT-PCR, underwent a $67 \%$ downregulation as compared to control mock-nucleofected cells, no inhibition of differentiation was detected by either clonogenic assay or phenotype analysis (data not shown). These results were quite expected in light of the consideration that the molecular control of monocyte commitment is a redundant process in which other transcription factors, as HOX-A $10^{6}$ and VDR, ${ }^{11}$ have been demonstrated to play a fundamental role in similar CD34 + based experimental models. In addition, the loss of MafB function could be probably compensated by other Maf family proteins exhibiting an upregulated expression during mono-macrophage differentiation of CD34 + hematopoietic progenitors. ${ }^{10}$ Experiments of multiple gene inactivation will help to verify these hypotheses.

\section{Genetic program activated by MafB transduction in CD34 + hematopoietic progenitors}

To determine MafB dependent genetic program, we analyzed the mRNA expression profile induced by retroviral 


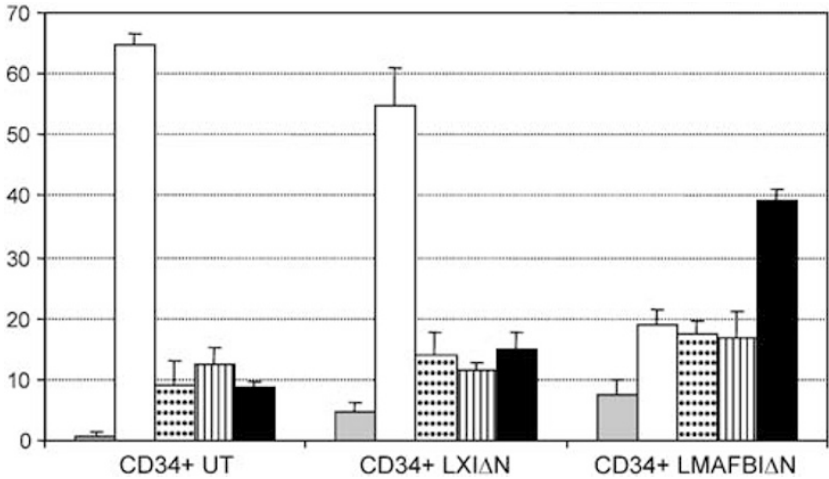

Figure 6 Clonogenic capacity of MafB transduced CD34 + cells. CD34 + hematopoietic progenitors, transduced with the $L M a f B I \Delta N$ and the $L X I \Delta N$ retroviral vectors at day 3 of liquid culture, were NGFR purified, plated 2 days postinfection in methylcellulose medium in the presence of cytokines and scored after 14 days as CFU-GEMM (gray bars), BFU-E/CFU-E (white bars), CFU-GM (dotted bars), CFU-G (dashed bars) and CFU-M (black bars). Untransduced control cells (UT) maintained in the same experimental conditions were also analyzed

transduction of this transcription factor in CD34 + cells, using the Affymetrix microarray methodology. Microarray analysis was performed at day 7 of liquid culture (4 days post-transduction) based on the observation that this timing disclosed the best differentiation effect at least in terms of CD14 antigen expression (see Figure 5). Cells transduced with the empty $\mathrm{LXI} \Delta \mathrm{N}$ vector were used as control. The results obtained disclosed the upregulation of 223 genes and the downregulation of 57 genes (SLR $\geq+1$ and $\leq-1$, respectively). GO mapping analysis, performed according to the biological process modality, evidenced the induction of genes belonging to the defense/immunity category and a downregulated expression of genes involved in hemoglobin assembly/ oxygen transport, consistently with the biological effects observed in liquid and semisolid cultures. Detailed analysis of the investigated expression profiles indicated that the majority of upregulated genes could be classified into the following functional categories: transcription factors, differentiation markers (CD antigens, granule proteins), secretory proteins, surface receptors, apoptosis and cell cycle related genes.

Among transcription factors upregulated by MafB transduction, we could detect genes belonging to the Maf (c-Maf), ${ }^{8}$ AP-1 (FosL2), ${ }^{14}$ PPAR ( $\delta$ member), ${ }^{10}$ MiT (MitF),${ }^{15}$ SMAD (SMAD3), ${ }^{16}$ and Hox (HLX1) ${ }^{17}$ families, already described for their capacity to regulate monocyte differentiation/activation or other aspects of hematopoiesis (Figure 7).

As expected, MafB transduction resulted in a clear induction of the CD14 and CD163 monocyte-specific differentiation antigens. More in general, virtually all CD antigens upregulated by MafB expression have been previously detected on monocyte/macrophage surface where they mediate a variety of biological functions related to innate and specific immunity and particularly: cell adhesion (CD44, CD51, CD54), phagocytosis of opsonized antigens (CD32, CD64), binding of bacterial and viral components (CD14, CD54, CD209), scavenging of inflammatory molecules (CD36L2, CD163) and initiation of T cell-mediated immune responses (CD80, CD83, CD85, CD86, CD209) (for a detailed description of expression pattern and biological functions of the above-mentioned CD antigens, see www.ncbi.nlm.nih. gov/prow) (Figure 7).

Similarly, MafB transduced CD34 + cells exhibited an increased expression of genes coding for typical macrophage granule proteins (Cathepsins, RNAses) and metalloproteinases (MMPs), ${ }^{18,19}$ respectively involved in intracellular antigen degradation and extracellular matrix remodeling (Figure 7).

Consistently with these findings, all secretory proteins and surface receptors exhibiting an upregulated mRNA expression in MafB transduced CD34 + cells have been previously demonstrated to play a role in different aspects of inflammatory response such as leukocyte chemotaxis (CCLs, IL-8), ${ }^{18}$ recognition and scavenging of bacterial products (MRCs, ${ }^{18}$ STAB $1^{20}$ ), secretion and binding of complement components (C1Q, C1QR1, IF, C3AR1, C5R1), production and activation of monocytes (GM-CSFR, M-CSFR, IL13RA1, IFNGR, TNFRSF14, IL1R2, TREM2, Z39IG, IL1RN) ${ }^{18}$ (Figure 7).

Analysis of cell cycle regulators revealed an increased mRNA expression of the GADD45B growth arrest/proapoptotic gene ${ }^{21}$ and a clear downregulation of universally recognized proliferation markers as the c-myc proto-oncogene, ${ }^{22}$ the CDC25A phosphatase ${ }^{23}$ and the TFDP2 transcription factor $^{24}$ (Figure 7).

Among downregulated genes, we were also able to detect those coding for: transcription factors (EKLF), globin chains $(\alpha, \beta, \varepsilon, \delta)$, cytoskeleton proteins (ankirin, spectrin, adducin) and surface antigens (GpC, transferrin receptor, Rh antigen) typically associated with erythroid differentiation, confirming the capacity of MafB to inhibit erythroid commitment ${ }^{25}$ (Figure 7).

Transgene dosage analysis, performed 4 days posttransduction by quantitative real time PCR, evidenced that MafB transcript was only 2.7-fold more expressed in transduced CD34 + cells as compared to normal peripheral blood (PB) monocytes, indicating that viral transduction in the adopted experimental conditions induces levels of MafB mRNA that are comparable to those observed in a physiological cell context (Figure 8). This finding excludes the possibility that microarray data obtained in the CD34+ cell model are the consequence of an abnormal MafB overexpression leading to non-specific activation of gene transcription.

\section{Discussion}

Physiologically occurring upregulation of specific transcription factors is a generally accepted mechanism to explain the commitment of HSCs along precise differentiation lineages and the subsequent maturation of committed progenitors. ${ }^{3}$ In this regard, a clear distinction has been established between transcription factors that are generically involved in the molecular control of hematopoietic cell differentiation and the so-called 'master' regulators, which are by themselves able to promote the lineage commitment of pluripotent HSCs or the lineage 'switching' of already committed progenitors. ${ }^{3,4}$ Additional properties of a master regulator are also 


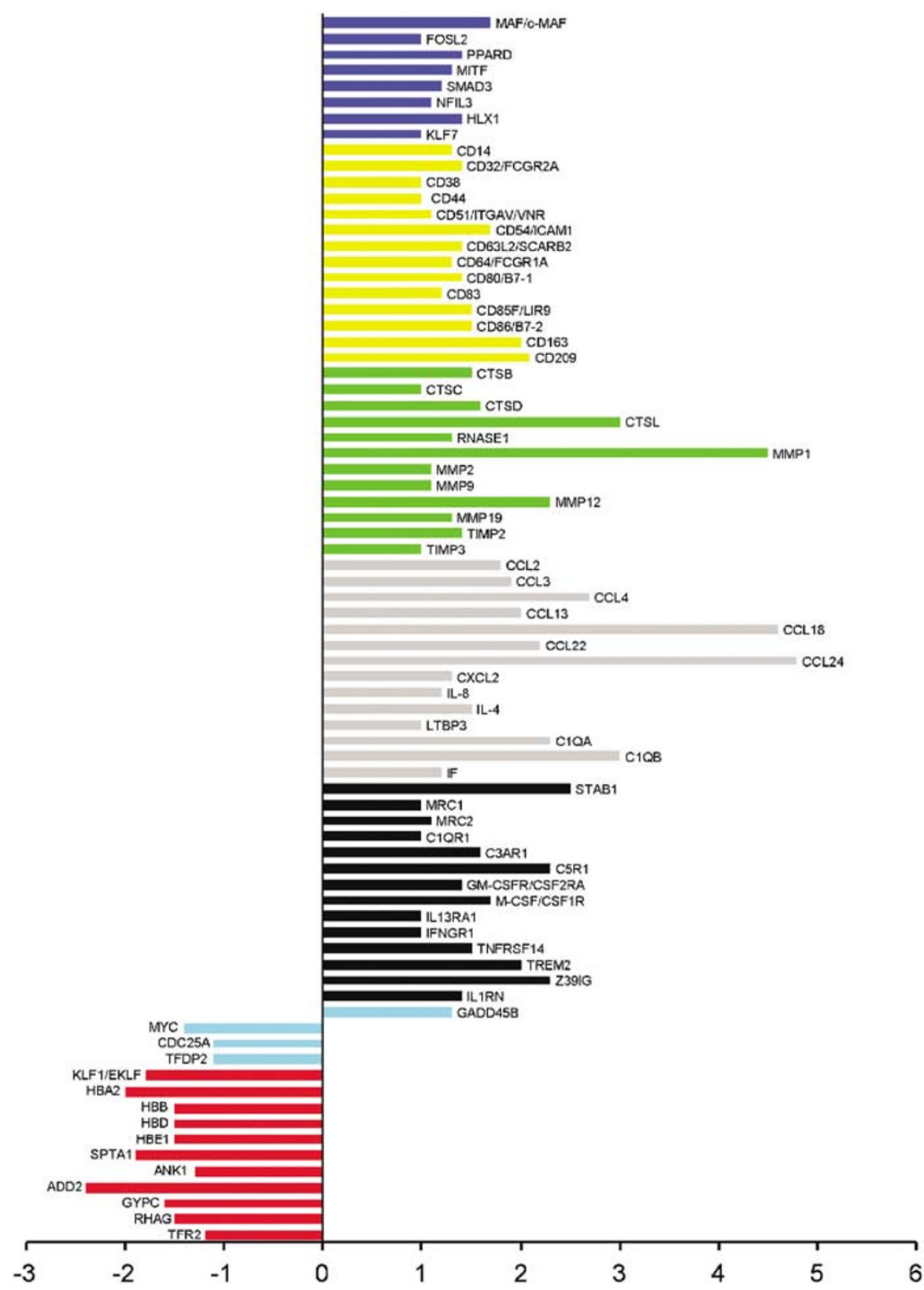

Figure 7 Gene expression profile induced by MafB transduction in CD34 + cells. Microarray analysis was performed at day 7 of liquid culture (4 days postinfection) on $\mathrm{CD} 34+$ cells transduced with the LMafBI $\Delta \mathrm{N}$ and LXI $\Delta \mathrm{N}$ retroviral vectors and NGFR purified. Changes in gene expression were assessed by comparison between cells transduced with the two analyzed vectors and reported on the $x$-axis of the shown histogram as signal log ratio (SLR) (fold change $=2^{\text {SLR }}$ ). Up-and downregulated genes (SLR $\geq+1$ and $\leq-1$, respectively) were classified in the following functional categories: transcription factors (blue bars), CD antigens (yellow bars), granule proteins (green bars), secretory proteins (gray bars), surface receptors (black bars), cell cycle related genes (light blue bars), erythroid associated genes (red bars). A list of selected genes induced by MafB is presented and indicated by Gene Symbols (complete/extended designation and description of each gene is available in the OMIM website). See Materials and Methods for details

represented by the capacity to inhibit the commitment towards alternative maturation lineages and the ability to transactivate and cooperate other transcription factors controlling the same differentiation lineage. ${ }^{4}$ Knockout and transgenic mice experiments have allowed the identification of a large number of this last category of transcription factors, the most important being represented by GATA-1 for erythroid differentiation, PU-1 for myeloid commitment and $\mathrm{C}-\mathrm{EBP} \alpha$ for granulocyte maturation. ${ }^{4}$ In addition, when human hematopoiesis is the investi- gated process, viral-mediated expression of a given transcription factor in CD34 + hematopoietic progenitors and leukemic cell lines can be the suitable experimental model to define its role in lineage commitment and terminal maturation, respectively. By using this approach, the HOXA10 gene has been recently demonstrated to induce the monocyte differentiation of $\mathrm{U} 937$ monoblasts $^{26}$ and CB CD34 + cells. ${ }^{6}$ Similarly, MafB transcription factor was reported to promote the monocytic commitment of Myb-Ets 


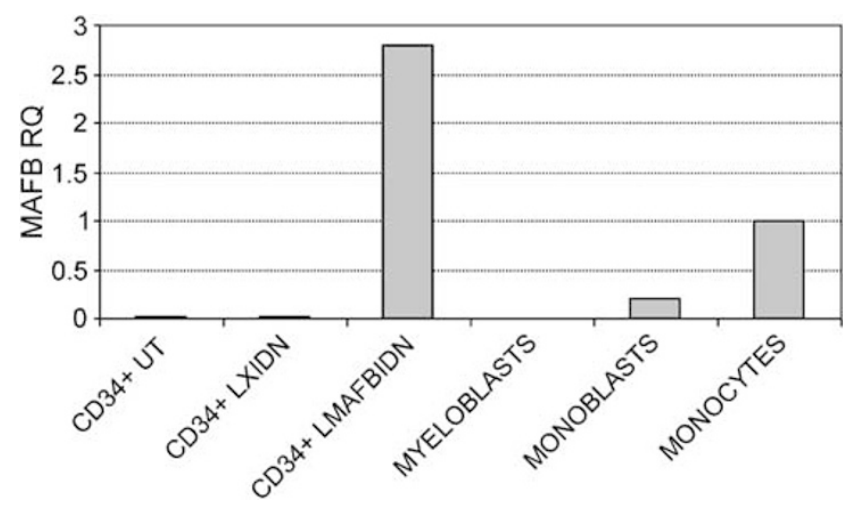

Figure 8 Quantitative RT-PCR analysis of MafB transcript in transduced $\mathrm{CD} 34+$ cells. Levels of MafB transcript were analyzed 7 days postinfection, by quantitative real time PCR, in untransduced (UT), 'empty' vector transduced $(\mathrm{LXI} \Delta \mathrm{N})$ and MafB transduced (LMafBI $\Delta \mathrm{N}) \mathrm{CD} 34+$ cells and compared to those of primary $\mathrm{CD} 34+$ derived myeloblasts and monoblasts and normal circulating monocytes. Differences in expression levels are reported on $y$-axis as relative quantity $(\mathrm{RQ})$ normalized using monocyte signal as calibrator

transformed avian hematopoietic progenitors, ${ }^{7}$ although, to date, this observation has not been confirmed in normal human hematopoiesis. Our data evidenced that MafB is selectively expressed in monocyte maturation lineage either in normal or leukemic hematopoietic cell populations. In addition, retroviral transduction of MafB, in experimental conditions that substantially mimicked its natural expression pattern, induced the mono-macrophage differentiation of monoblastic U937 and THP1 cell lines and CD34 + hematopoietic stem/progenitor cells as assessed by flow cytometry (CD14, CD11b) and morphological analysis. The extent of differentiation was more pronounced in the latter as compared to the former cells. This discrepancy can be ascribed to the higher differentiation potentiality of CD34 + cells and/or to the maturation arrest of U937 and THP1 leukemic cells. It is worth considering that in both cell contexts immune phenotype underestimated the differentiation effect in comparison with morphological analysis. The induction of monocyte commitment, observed in CD34 + cells upon MafB transduction, was accompanied by a massive inhibition of erythroid progenitor development and a downregulated expression of erythroid related genes, as assessed by clonogenic assay and microarray analysis, respectively. Interestingly, this unbalance of hematopoietic lineage commitment exactly reproduced the effect promoted, on the same cells, by stimulation with physiological levels of Vitamin $D 3^{11}$ or retroviral transduction of the Hox-A10 gene, ${ }^{27}$ suggesting a probable cooperation among the corresponding intracellular pathways. To determine MafB dependent genetic program, we performed a set of gene expression profiling experiments on MafB transduced CD34 + hematopoietic stem/progenitor cells. GO mapping and detailed analysis of the considered expression profiles revealed that virtually all upregulated genes were strictly related to mono-macrophage differentiation and functional activation. In fact, beside the already mentioned CD14 and CD11b antigens, MafB induced the expression of other typical markers of monocyte differentiation/activation such as CD163, cathepsin B and D, RNAse A 1, MMP2 and 9, CCL2 and 3 and others. ${ }^{18}$ In addition, retroviral transduction of this transcription factor leaded to increased mRNA levels of receptors promoting macrophage activation in response to cytokine stimulation (M-CSF, IL13, IFN $\gamma$ ). ${ }^{18}$ Consistently with these findings, MafB also upregulated scavenger receptors involved in internalization and degradation of Gram + and Gram - bacteria such as mannose receptors $^{18}$ (MRC1 and 2) and STAB1. ${ }^{20}$ Interestingly, this genetic program included the induction of negative regulators of monocyte activation as the $\mathrm{Z3} \mathrm{IG} \mathrm{G}^{28} \mathrm{Ig}$-like receptor and the IL-1 receptor antagonist ${ }^{29}$ (IL1RN). Almost all transcription factors upregulated by MafB in CD34 + cells have been demonstrated to play a role or to be somehow involved in the regulation of mono-macrophage differentiation. In particular: (1) Fos family members, ${ }^{14}$ responsible for the assembly of the AP-1 transcription complex together with Jun proteins, and the c-Maf gene ${ }^{9}$ have been reported to induce a partial macrophage maturation of several myeloid cell lines (M1, U937, HL60); (2) the PPAR $\delta$ nuclear receptor is a transactivator of the CCL2/MCP1 chemokine in macrophages; ${ }^{30}$ (3) MiT family transcription factors are macrophage-restricted, even if very little is known about their target genes; ${ }^{15}$ (4) SMAD3 is a transcription factor responsible for the TGF $\beta$ pathway intracellular effects, ${ }^{16}$ previously demonstrated to promote growth arrest and monocyte differentiation at least in cell line models (U937); ${ }^{31}$ (5) NFIL3 ${ }^{32}$ drives the transcription of IL-3, a cytokine involved in the regulation of granulo-monocytopoiesis. ${ }^{18}$ The upregulated expression of such transcription factors may consequently provide the molecular basis to explain the effects played by MafB in the control of mono-macrophage differentiation. Since Fos and large Maf family proteins are well-known MafB dimerization partners, ${ }^{33}$ it is conceivable that MafBmediated induction of mono-macrophage differentiation occurs through the interaction with members belonging to this transcription factor family. It has to be pointed out that virtually all transcription factors mentioned above, or at least other members of the same families, exhibited an upregulated expression in CD34+ derived monocyte precursors as compared to granulocyte precursors,${ }^{10}$ underlining their crucial importance in the regulation of monocyte commitment.

As expected MafB-mediated differentiation was coupled with a remarkable inhibition of cell growth. Again microarray analysis provided a plausible explanation for this biological effect evidencing the induction of proapoptotic genes and the downregulation of cell cycle related genes. It is worth consider that normal monoblasts deriving from spontaneous, cytokineinduced, differentiation of CD34 + cells 'in vitro' exhibit a strong upregulation of MafB expression and are characterized by a similar combination between monocyte maturation and early proliferation arrest. $^{10}$

Although we are not able to state that upregulated genes are direct target of MafB, and proper assays such as chromatin immuno-precipitation are required to address this issue, taken together our data provide a clear demonstration that the investigated transcription factor is able to promote mono-macrophage differentiation acting at different levels of hematopoiesis, as evidenced by the experiments performed in CD34 + cells and leukemic monoblasts, respectively. These data allow to conclude that MafB transcription factor behaves as a master regulator of monocytic commitment in a human model of hematopoiesis. 


\section{Materials and Methods}

\section{Hematopoietic cell lines}

K562, KG1a, KG1, KASUMI1, HL60, NB4, THP1 and U937 cell lines were obtained from ATCC (Rockville, MD, USA) and cultured in RPMI1640 medium (Euroclone, Devon, UK), supplemented with 10\% heat inactivated fetal bovine serum (Biowhittaker, Walkersville, MD, USA) and $1 \mathrm{mM}$ L-glutamine (Euroclone).

\section{Primary hematopoietic cells}

Human CD34 + cells were purified from umbilical CB samples as already described, ${ }^{10,11}$ and seeded $\left(5-10 \times 10^{4} / \mathrm{ml}\right)$ in Iscove's modified Dulbecco's medium (IMDM) (Euroclone) containing 10\% human serum (Biowhittaker) and early acting human hematopoietic cytokines: $50 \mathrm{ng} / \mathrm{ml}$ stem cell factor (SCF) and Flt3-ligand (Flt3-l), $20 \mathrm{ng} / \mathrm{ml}$ thrombopoietin (TPO), $10 \mathrm{ng} / \mathrm{ml}$ interleukin-11 (IL-11), interleukin-6 (IL-6) and interleukin-3 (IL-3) (R\&D Systems, Minneapolis, MN, USA). These experimental conditions normally promote a mixed granulo-monocyte differentiation of $\mathrm{CD} 34+$ cells that is generally achieved within 14 days from plating. ${ }^{11,12,34}$

Normal human monocytes were selected from Ficoll separated PB mononuclear cells of adult samples by using magnetic microbeads conjugated with mouse monoclonal (Mo) anti-human CD14 antibody (Ab) (Miltenyi Biotec, Auburn, CA, USA). Human lymphocytes were obtained as negative fraction of this last purification procedure. Human granulocytes were initially collected from cell pellets obtained by Ficoll separation of PB samples. Neutrophils were then purified using magnetic microbeads conjugated to mouse Mo anti-human CD16 Ab (Miltenyi Biotec). Purity of purified primary cell populations, determined by flow cytometry and morphological analysis (May-Grunwald-Giemsa staining), always exceeded $95 \%$.

Myeloblasts, ${ }^{10}$ monoblasts ${ }^{10}$ and megakaryoblasts ${ }^{35}$ were generated by 'in vitro' culture of CB CD34 + hematopoietic progenitors performed as already described. Human normal erythroblasts were obtained by culturing CD34 + cells in IMDM supplemented with 10\% BIT (Stem cells Technologies, Vancouver, BC, Canada), $50 \mathrm{ng} / \mathrm{ml} \mathrm{SCF}$ and $5 \mathrm{U} / \mathrm{ml}$ Erythropoietin (R\&D Systems) as previously described. ${ }^{34}$

\section{Retroviral vector construction and packaging}

A full-length MafB CDNA was generated by RT-PCR performed on total RNA extracted from $1 \alpha, 25$ di-hydroxy-vitamin $D_{3}$ (VD)-stimulated CB $\mathrm{CD} 34+$ cells using MafB DP and MafB $\mathrm{RP}_{1}$ primers (Supplementary Table 1) and a proof-reading thermostable DNA polymerase. The amplified fragment was then inserted in the pCR2.1 T/A cloning vector (Invitrogen, Carlsbad, CA, USA) generating the pCR2.1MafB plasmid, where it was fully sequenced to exclude polymerase-induced mutations.

A MafB containing fragment, amplified from this plasmid using modified oligonucleotide primers (MafB-DP-Not and MafB-RP-Bgl, see Supplementary Table 1), was subsequently inserted into the Notl/Bgll digested pFlagCMV-2 plasmid where the Flag epitope was fused to the $\mathrm{N}$-terminus of MafB cDNA. This last sequence was in turn amplified using distinct primers containing the EcoRI site (MafB-FLAG-DP-Eco and MAFB-RPEco primers; Supplementary Table 1).

MafB and FlagMafB cDNAs were then EcoRl excised and cloned in the $E c o R I$ site of $L X I \Delta N$ vector ${ }^{36}$ resulting in the construction of $L M a f B I \Delta N$ and LFLAGMafBI $\Delta \mathrm{N}$ retroviral vectors, respectively.

Packaging lines for all the described constructs were generated by transinfection in the ecotropic Phoenix and amphotropic GP + envAm12 cells, as previously described. ${ }^{34}$ Viral titers were assessed by flow cytometry analysis of $\triangle \mathrm{LNGFR}$ expression percentage upon infection of U937 and THP1 cells, and CD34 + hematopoietic progenitors.

\section{Hematopoietic cell transduction and purification}

U937 and THP1 hematopoietic cell lines were transduced by two to three cycles of infection ( $4 \mathrm{~h}$ each) with viral supernatant in the presence of polybrene $(8 \mu \mathrm{g} / \mathrm{ml})$. Transduced U937 cells were sorted using a purified mouse anti-human p75-NGFR MoAb (PharMingen, San Diego, CA, USA) and M-450 goat anti-mouse immuno-magnetic beads (Dynal, Oslo, Norway) following the manufacturer's guidelines.

Human CB CD34 + progenitors, preactivated for $48 \mathrm{~h}$ in the already described liquid culture conditions, were transduced by two cycles of infection (12 $\mathrm{h}$ each) with viral supernatant on retronectin-coated plates $\left(10 \mathrm{~g} / \mathrm{cm}^{2}\right)$. Purification of transduced CD34 + cells was achieved by an immunomagnetic procedure using mouse anti-human p75-NGFR MoAb and tiny fluorescence activated cell sorter-compatible magnetic nanoparticles in a column-free magnetic system (EasySep ${ }^{\mathrm{TM}}$ 'Do-It-Your Self' Selection Kit (Stem cells Technologies)). To perform gene inactivation experiments, $5 \mu \mathrm{g}$ of siRNA oligonucleotide targeting MafB (Dharmacon, Inc., Lafayette, Colorado, USA) was transfected into CB CD34 + cells by means of the Nucleofector methodology (Amaxa Biosystems $\mathrm{GmbH}$, Cologne, Germany), according to the manufacturer's guidelines. Culture of nucleofected cells was carried out as described above for retroviral transduction experiments.

\section{Flow cytometry analysis of transduced cells}

Transduction efficiency of U937, THP1 and CD34 + cells was monitored by flow cytometry analysis (Epics $\mathrm{XL}$, Coulter) of $\triangle \mathrm{LNGFR}$ expression using a murine anti-human p75-NGFR MoAb conjugated with biotin (BD Biosciences, San Diego, CA, USA) and streptavidin conjugated to R-Phycoerythrin-Cy5 (RPE-Cy5) (DAKO A/S, Copenhagen, Denmark). The extent of monocyte differentiation was monitored using a mouse antiCD14 MoAb conjugated to fluorescein isothiocyanate (FITC) and a mouse PE-conjugated anti-CD11b MoAb (both from BD Biosciences). Antibodies incubations were carried out as described. ${ }^{11}$

\section{CFC assay}

Untransduced, $\mathrm{LXI} \Delta \mathrm{N}$ transduced and $\mathrm{LMafBI} \Delta \mathrm{N}$ transduced $\mathrm{CD} 34+$ cells were NGFR purified at day 5 of liquid culture and plated in a complete methylcellulose medium (MethoCult ${ }^{\mathrm{TM}} \mathrm{H} 4434$, Stem Cell Technologies) as already described. ${ }^{11}$ Colonies were scored 14 days after plating as erythroid (burst forming unit-erythroid (BFU-E) + colony forming-unit (CFU-E)), CFU-GM, CFU-G or GFU-M.

\section{RT-PCR and Northern blot analysis}

Total cellular RNA from cell lines and primary hematopoietic cells was isolated by means of the guanidinium-cesium chloride centrifugation technique ${ }^{37}$ and digested with RQ1-DNAse (Promega, Madison, WI, USA) as already described. ${ }^{38}$ RNA integrity and concentration was then assessed by the Bio-Analyzer technique (Applied Biosystem, Foster City, CA, USA). Expression of MafB at the mRNA level was assessed using reverse transcriptase-polymerase chain reaction (RT-PCR), performed as previously described, ${ }^{38}$ using MafB DP and MafB $\mathrm{RP}_{2}$ oligonucleotide primers (Supplementary Table 1). Normalization of the amplified samples was obtained by the glyceraldehydes 3-phosphate dehydrogenase 
(GAPDH) 'housekeeping' gene as previously described..$^{11}$ Northern blot analysis was performed as described. ${ }^{36,39}$

\section{Quantitative real time PCR (QRT-PCR)}

Total RNA (100 ng) was reverse transcribed using High Capacity cDNA Archive Kit (Applied Biosystems) according to the manufacturer's instructions. QRT-PCR was then performed with an ABI PRISM 7900 sequence detection system (Applied Biosystems) to quantify the relative levels of MafB mRNA in the samples. Primers and probe for MafB amplification were designed, using the File Builder Software (Applied Biosystems), in the 5' region of MafB coding sequence. Quantitation was performed by amplifying GAPDH mRNA as endogenous control using a strategy designed by Applied Biosystems. Each cDNA sample was run in triplicate for MafB (target) and GAPDH (endogenous control) in $50 \mu \mathrm{l}$ reaction volume using Taqman Universal PCR Master Mix (Applied Biosystems). Thermal cycling was started with an initial denaturation at $50^{\circ} \mathrm{C}$ for $2 \mathrm{~min}$ and $95^{\circ} \mathrm{C}$ for $10 \mathrm{~min}$, followed by 40 cycles of $15 \mathrm{~s}$ at $95^{\circ} \mathrm{C}$ and $1 \mathrm{~min}$ at $60^{\circ} \mathrm{C}$. Statistical analysis of the quantitative real time PCR was obtained using the $\left(2^{-\Delta \Delta C t}\right)$ method, ${ }^{40}$ which calculates relative changes in gene expression of the target gene normalized to the endogenous control and relative to a calibrator (monocytes) which serves as control.

\section{Nuclear extract preparation and Western blot analysis}

Nuclear extracts of untransduced and LMafBI $\Delta \mathrm{N}$ transduced U937 cells were obtained as previously reported with minor modifications. ${ }^{11}$ Briefly, $30 \mu \mathrm{g}$ of nuclear extracts of the analyzed cell populations were loaded onto $15 \%$ sodium dodecyl sulphate-polyacrylamide gel electrophoresis and blotted as described. ${ }^{11}$ Membranes were preblocked in blocking solution, supplemented with $4 \mathrm{mg} / \mathrm{ml}$ normal serum and $10 \%$ blocking reagent (Roche Diagnostics, Mannheim, Germany), for $1 \mathrm{~h}$ at room temperature and then incubated with a 1:500 dilution of the mouse MoAb anti-FLAG M2 (Sigma Chemical Co., St. Louis, MO, USA), followed by a 1-h incubation at room temperature with a secondary Ab goat anti-mouse conjugated to horse-radish peroxidase 1:10000 (Santa Cruz Biotechnology, Inc., Santa Cruz, CA, USA). Detection was carried out by using the BM Chemiluminescence Blotting Substrate (Roche Diagnostics).

\section{Biotin labeled transcription, Genechip hybridization and microarray data analysis}

RNA pools $(5 \mu \mathrm{g})$ of $\mathrm{LXI} \Delta \mathrm{N}$ and $\mathrm{LMafBI} \Delta \mathrm{N}$ transduced CD34 + cells obtained from three different experiments were converted in labelled cRNA and then hybridized to Affymetrix HG-U133A GeneChip arrays as already described. ${ }^{10}$ Images obtained by scanning chips of untransduced, $\mathrm{LXI} \Delta \mathrm{N}$ and $\mathrm{LMafBI} \Delta \mathrm{N}$ transduced $\mathrm{CD} 34$ + cells were processed using the Affymetrix Microarray Analysis Suite 5.0 (MAS 5.0). This software allows to assign an 'Absolute Call' ('Absent' or 'Present'), to quantify mRNA expression levels in terms of a 'Signal' value and to attribute a 'Change' 'Increased' (I), 'Decreased' (D) or 'Not Changed' (NC) to each transcript in the comparison between two analyzed cell populations. Fold changes of the analyzed mRNAs observed comparing the considered sample (LMafBI $\Delta \mathrm{N}$ transduced cells) to the baseline ( $\mathrm{LXI} \Delta \mathrm{N}$ transduced cells) were then expressed as signal log ratio (SLR) (fold change $=2^{\mathrm{SLR}}$ ).

The Gene Ontology Mining Tool Affymetrix (www.affymetrix.com/ netaffx) database was used to cluster annotated U133A genes for functional categories. Gene categories were chosen by browsing the three main terms of the ontology vocabulary: molecular function, biological process and cellular component

Clustering analysis of primary hematopoietic cell populations was performed by means of the GeneSpring software 6.2 (Silicon Genetics, www.silicongenetics.com).

\section{Acknowledgements}

This work has been supported by a grant from AIRC 2004 and from MURST-Cofin 2003

\section{References}

1. Metcalf D (1989) The molecular control of cell division, differentiation commitment and maturation in haemopoietic cells. Nature 339: 27-30

2. Payne KJ and Crooks GM (2002) Human hematopoietic lineage commitment. Immunol. Rev. 187: 48-64

3. Tenen DG, Hromas R, Licht JD and Zhang DE (1997) Transcription factors, normal myeloid development, and leukemia. Blood 90: 489-519

4. Orkin SH (2000) Diversification of haematopoietic stem cells to specific lineages. Nat. Rev. Genet. 1: 57-64

5. Graf T (2002) Differentiation plasticity of hematopoietic cells. Blood 99: 3089-3101

6. Taghon T, Stolz F, De Smedt M, Cnockaert M, Verhasselt B, Plum J and Leclercq G (2002) HOX-A10 regulates hematopoietic lineage commitment: evidence for a monocyte-specific transcription factor. Blood 99: 1197-1204

7. Kelly LM, Englmeier U, Lafon I, Sieweke MH and Graf T (2000) MafB is an inducer of monocytic differentiation. EMBO J. 19: 1987-1997

8. Blank V and Andrews NC (1997) The Maf transcription factors: regulators of differentiation. Trends Biochem. Sci. 22: 437-441

9. Hegde SP, Zhao J, Ashmun RA and Shapiro LH (1999) c-Maf induces monocytic differentiation and apoptosis in bipotent myeloid progenitors. Blood 94: 1578-1589

10. Montanari M, Gemelli C, Tenedini E, Zanocco MT, Vignudelli T, Siena M, Zini R, Salati S, Chiossi G, Taglifico E, Manfredini R, Grande A and Ferrari S (2005) Correlation between differentiation plasticity and mRNA expression profiling of CD34+-derived. Cell Death Differ. 12: 1588-1600

11. Grande A, Montanari M, Tagliafico E, Manfredini R, Marani TZ, Siena M, Tenedini E, Gallinelli A and Ferrari S (2002) Physiological levels of 1alpha, 25 dihydroxyvitamin D3 induce the monocytic commitment of CD34+ hematopoietic progenitors. J. Leukoc. Biol. 71: 641-651

12. Piacibello W, Sanavio F, Garetto L, Severino A, Dane A, Gammaitoni $L$ and Aglietta M (1998) Differential growth factor requirement of primitive cord blood hematopoietic stem cell for self-renewal and amplification vs proliferation and differentiation. Leukemia 12: 718-727

13. Naif HM, Li S, Ho-Shon M, Mathijs JM, Williamson P and Cunningham AL (1997) The state of maturation of monocytes into macrophages determines the effects of IL-4 and IL-13 on HIV replication. J. Immunol. 158: 501-511

14. Lord KA, Abdollahi A, Hoffman-Liebermann B and Liebermann DA (1993) Proto-oncogenes of the fos/jun family of transcription factors are positive regulators of myeloid differentiation. Mol. Cell Biol. 13: 841-851

15. Hershey CL and Fisher DE (2004) Mitf and Tfe3: members of a b-HLH-ZIP transcription factor family essential for osteoclast development and function. Bone 34: 689-696

16. Yanagisawa J, Yanagi $Y$, Masuhiro $Y$, Suzawa M, Watanabe M, Kashiwagi $K$, Toriyabe T, Kawabata M, Miyazono K and Kato S (1999) Convergence of transforming growth factor-beta and vitamin $D$ signaling pathways on SMAD transcriptional coactivators. Science 283: 1317-1321

17. Kehrl JH and Deguchi Y (1993) Potential roles for two human homeodomain containing proteins in the proliferation and differentiation of human hematopoietic progenitors. Leuk. Lymphoma 10: 173-176

18. Weinberg JB (2004) Mononuclear Phagocytes. Part III. The Normal Hematologic System (Philadelphia, PA: Lippincott Williams \& Wilkins) pp. 377-414 (Chapter 16).

19. Borregaard N and Cowland JB (1997) Granules of the human neutrophilic polymorphonuclear leukocyte. Blood 89: 3503-3521 
20. Adachi $H$ and Tsujimoto $M$ (2002) FEEL-1, a novel scavenger receptor with in vitro bacteria-binding and angiogenesis-modulating activities. J. Biol. Chem. 277: 34264-34270

21. Carrier F, Smith ML, Bae I, Kilpatrick KE, Lansing TJ, Chen CY, Engelstein M Friend SH, Henner WD and Gilmer TM (1994) Characterization of human Gadd45, a p53-regulated protein. J. Biol. Chem. 269: 32672-32677

22. Bouchard C, Thieke K, Maier A, Saffrich R, Hanley-Hyde J, Ansorge W, Reed S, Sicinski P, Bartek J and Eilers M (1999) Direct induction of cyclin D2 by Myc contributes to cell cycle progression and sequestration of p27. EMBO J. 18: 5321-5333

23. Furnari B, Rhind N and Russell $P$ (1997) Cdc25 mitotic inducer targeted by chk1 DNA damage checkpoint kinase. Science 277: 1495-1497

24. Zhang W, Zhang J, Kornuc M, Kwan K, Frank R and Nimer SD (1995) Molecular cloning and characterization of NF-IL3A, a transcriptional activator of the human interleukin-3 promoter. Mol. Cell Biol. 15: 6055-6063

25. Koury MJ, Sawyer ST and Brandt SJ (2002) New insights into erythropoiesis. Curr. Opin. Hematol. 9: 93-100

26. Rots NY, Liu M, Anderson EC and Freedman LP (1998) A differential screen for ligand-regulated genes: identification of HoxA10 as a target of vitamin D3 induction in myeloid leukemic cells. Mol. Cell Biol. 18: 1911-1918

27. Buske C, Feuring-Buske M, Antonchuk J, Rosten P, Hogge DE, Eaves CJ and Humphries RK (2001) Overexpression of HOXA10 perturbs human lymphomyelopoiesis in vitro and in vivo. Blood 97: 2286-2292

28. Walker MG (2002) Z39lg is co-expressed with activated macrophage genes. Biochim. Biophys. Acta. 1574: 387-390

29. Dayer JM (2002) Evidence for the biological modulation of IL-1 activity: the role of IL-1Ra. Clin. Exp. Rheumatol. 20 (Suppl 27): S14-S20

30. Plutzky J (2003) Medicine. PPARs as therapeutic targets: reverse cardiology? Science 302: 406-407

31. Zawel L, Dai JL, Buckhaults P, Zhou S, Kinzler KW, Vogelstein B and Kern SE (1998) Human Smad3 and Smad4 are sequence-specific transcription activators. Mol. Cell. 1: 611-617
32. Zhang $Y$ and Chellappan SP (1995) Cloning and characterization of human DP2, a novel dimerization partner of E2F. Oncogene 10: 2085-2093

33. Kataoka K, Noda M and Nishizawa M (1994) Maf nuclear oncoprotein recognizes sequences related to an $\mathrm{AP}-1$ site and forms heterodimers with both Fos and Jun. Mol. Cell Biol. 14: 700-712

34. Grande A, Piovani B, Aiuti A, Ottolenghi S, Mavilio F and Ferrari G (1999) Transcriptional targeting of retroviral vectors to the erythroblastic progeny of transduced hematopoietic stem cells. Blood 93: 3276-3285

35. Tenedini E, Fagioli ME, Vianelli N, Tazzari PL, Ricci F, Tagliafico E, Ricci P, Gugliotta L, Martinelli G, Tura S, Baccarani M, Ferrari S and Catani L (2004) Gene expression profiling of normal and malignant CD34-derived megakaryocytic cells. Blood 104: 3126-3135

36. Urbinati F, Lotti F, Facchini G, Montanari M, Ferrari G, Mavilio F and Grande A (2005) Competitive engraftment of hematopoietic stem cells genetically modified with a truncated erythropoietin receptor. Hum. Gene. Ther. 16: 594-608

37. MacDonald RJ, Swift GH, Przybyla AE and Chirgwin JM (1987) Isolation of RNA using guanidinium salts. Methods Enzymol. 152: 219-227

38. Grande A, Manfredini R, Tagliafico E, Balestri R, Pizzanelli M, Papa S, Zucchini P, Bonsi L, Bagnara G, Torelli U and Ferrari S (1995) All-transretinoic acid induces simultaneously granulocytic differentiation and expression of inflammatory cytokines in HL-60 cells. Exp. Hematol. 23: 117-125

39. Ferrari S, Tagliafico E, Ceccherelli G, Selleri L, Calabretta B, Donelli A Temperani P, Sarti M, Sacchi S, Emilia G, Torelli G and Torelli U (1989) Expression of the myeloperoxidase gene in acute and chronic myeloid leukemias: relationship to the expression of cell cycle-related genes. Leukemia 3: 423-430

40. Livak KJ and Schmittgen TD (2001) Analysis of relative gene expression data using real-time quantitative PCR and the 2(-Delta Delta C(T)) Method. Methods 25: $402-408$

Supplementary Information accompanies the paper on Cell Death and Differentiation website (http://www.nature.com/cdd) 